\title{
Spatial distribution of water-active soil layer along the south-north transect in the Loess Plateau of China
}

\author{
ZHAO Chunlei ${ }^{1,2}$, SHAO Ming'an ${ }^{1,2,3}$, JIA Xiaoxu ${ }^{1,2}$, HUANG Laiming ${ }^{1,2}$, ZHU Yuanjun Z $^{3 *}$ \\ ${ }^{1}$ Key Laboratory of Ecosystem Network Observation and Modeling, Institute of Geographical Sciences and Natural Resources \\ Research, Chinese Academy of Sciences, Beijing 100101, China; \\ ${ }^{2}$ College of Resources and Environment, University of Chinese Academy of Sciences, Beijing 100190, China; \\ ${ }^{3}$ State Key Laboratory of Soil Erosion and Dryland Farming on the Loess Plateau, Northwest A\&F University, Yangling 712100, \\ China; \\ ${ }^{4}$ College of Natural Resources and Environment, Northwest A\&F University, Yangling 712100, China
}

\begin{abstract}
Soil water is an important composition of water recycle in the soil-plant-atmosphere continuum. However, intense water exchange between soil-plant and soil-atmosphere interfaces only occurs in a certain layer of the soil profile. For deep insight into water active layer (WAL, defined as the soil layer with a coefficient of variation in soil water content $>10 \%$ in a given time domain) in the Loess Plateau of China, we measured soil water content (SWC) in the $0.0-5.0 \mathrm{~m}$ soil profile from 86 sampling sites along an approximately 860-km long south-north transect during the period 2013-2016. Moreover, a dataset contained four climatic factors (mean annual precipitation, mean annual evaporation, annual mean temperature and mean annual dryness index) and five local factors (altitude, slope gradient, land use, clay content and soil organic carbon) of each sampling site was obtained. In this study, three WAL indices (WAL$\mathrm{T}$ (the thickness of WAL), WAL-CV (the mean coefficient of variation in SWC within WAL) and WALSWC (the mean SWC within WAL)) were used to evaluate the characteristics of WAL. The results showed that with increasing latitude, WAL-T and WAL-CV increased firstly and then decreased. WAL-SWC showed an opposite distribution pattern along the south-north transect compared with WAL-T and WAL-CV. Average WAL-T of the transect was $2.0 \mathrm{~m}$, suggesting intense soil water exchange in the 0.0-2.0 $\mathrm{m}$ soil layer in the study area. Soil water exchange was deeper and more intense in the middle region than in the southern and northern regions, with the values of WAL-CV and WAL-T being $27.3 \%$ and $4.3 \mathrm{~m}$ in the middle region, respectively. Both climatic (10.1\%) and local (4.9\%) factors influenced the indices of WAL, with climatic factors having a more dominant effect. Compared with multiple linear regressions, pedotransfer functions (PTFs) from artificial neural network can better estimate the WAL indices. PTFs developed by artificial neural network respectively explained $86 \%, 81 \%$ and $64 \%$ of the total variations in WAL-T, WAL-SWC and WAL-CV. Knowledge of WAL is crucial for understanding the regional water budget and evaluating the stable soil water reserve, regional water characteristics and eco-hydrological processes in the Loess Plateau of China.
\end{abstract}

Keywords: water active layer; soil water content; redundancy analysis; pedotransfer function; artificial neural network; Loess Plateau

Citation: ZHAO Chunlei, SHAO Ming'an, JIA Xiaoxu, HUANG Laiming, ZHU Yuanjun. 2019. Spatial distribution of wateractive soil layer along the south-north transect in the Loess Plateau of China. Journal of Arid Land, 11(2): 228-240. https://doi.org//10.1007/s40333-019-0051-4

"Corresponding author: ZHU Yuanjun (E-mail: zhuyuanjun@foxmail.com)

Received 2017-12-11; revised 2018-09-20; accepted 2018-10-06

(C) Xinjiang Institute of Ecology and Geography, Chinese Academy of Sciences, Science Press and Springer-Verlag GmbH Germany, part of Springer Nature 2019 


\section{Introduction}

Soil water is not only a dominant factor for crop growth and vegetation restoration in semi-arid regions, but also a key element of water cycle in the soil-plant-atmosphere continuum (Hupet and Vanclooster, 2002; Heathman et al., 2009). For instance, at the soil-atmosphere interface, atmospheric water vapor recharges soil water through precipitation and soil water gets back into the atmosphere via evapotranspiration. At the soil-plant interface, soil water is consumed by plant root water uptake. Thus, precipitation, evapotranspiration and their interactions can cause fluctuations in soil water over time (Zhao et al., 2017a).

The overall variation in soil water can be described by the temporal dynamic of soil water content (SWC) (Biswas and Si, 2011; Hu et al., 2012) and the temporal variation of SWC which reflects the rate of soil water exchange at the soil-atmosphere and soil-plant interfaces (Heathman et al., 2009; Gao et al., 2015). The gradient of temporal dynamic of SWC is a good indicator for vegetation growth and a critical basis for SWC estimation, water resources assessment and hydrological model conceptualization (Brocca et al., 2010; Wang et al., 2015; Gasch et al., 2017). The temporal variation in SWC usually varies with topographic feature, soil physical property, vegetation type and climatic condition (Nyberg, 1996; Crave and Gascuel-Odoux, 1997; Mohanty et al., 2000). The dynamic of SWC along a soil profile is generally depth-dependent, weakening with increasing soil depth (Gao et al., 2015). Hupet and Vanclooster (2002) explored the temporal dynamic of SWC in a maize land on a plateau in Belgium and noted that there was a higher soil water variation in the surface soil layer. In a watershed in Ames, USA, Choi and Jacobs (2007) found that the temporal variation in SWC tends to decrease with increasing soil depth. Based on the gradient of the temporal dynamic of SWC, Gao et al. (2015) divided the soil profile into three layers in the Loess Plateau of China at a slope scale and pointed out that soil water fluctuated slightly with time below the depth of $1.6 \mathrm{~m}$. Thus, intense water exchange at the soil-plant and soilatmosphere interfaces only happens in a certain layer of the soil profile.

In the soil profile, water active layer (WAL) exhibits intense water exchange where soil water recharge and discharge occur. In this study, the WAL is defined as the layer with a coefficient of variation in SWC $>10 \%$ in a given time domain. The Loess Plateau is characterized by severe water shortage, thick loess deposit, intense soil erosion and fragile environments (Jia et al., 2013; Zhao et al., 2017a). Soils in the region originating from thick loess deposit generally have a high water storage capacity $(200-300 \mathrm{~mm} / \mathrm{m})(\mathrm{Li}, 1983$; Chen et al., 2008). The loess deposit usually has a thickness of 30-80 m (sometimes over $300 \mathrm{~m}$ ), which works like an active "reservoir" for regulating vegetation growth (Wang et al., 2012; Jia et al., 2019). WAL can be used to quantify the range and intensity of soil water exchange in the soil profile, and the knowledge of WAL deepens existing insights into the hydrologic process in the thick loss deposit for integrated water management and crop production in the Loess Plateau (Choi and Jacobs, 2007). Therefore, it is necessary to clarify the boundary and characteristics of WAL under various climatic and local conditions in the Loess Plateau. There is a conscious effort to determine the regional distribution of WAL and the related driving factors for developing sustainable vegetation restoration strategies in this region.

In this study, 18 SWC measurement occasions were conducted during 2013-2016 at 86 sampling sites along an approximately $860-\mathrm{km}$ long south-north transect across the typical Loess Plateau. Further, data of nine environmental factors (including four climatic factors and five local factors) were also collected to investigate the dominant driving factors of WAL in the study area. The specific objectives of the study were: (1) to investigate the spatial characteristics of the temporal variation in SWC and WAL in the typical Loess Plateau; (2) to determine the relative contribution of climatic and local factors to WAL; and (3) to develop reasonable pedotransfer functions (PTFs) to estimate the quantitative indices of WAL.

\section{Materials and methods}

\subsection{Study area}

The study was conducted in the typical Loess Plateau of China (Fig. 1), covering a total area of 
$0.43 \times 10^{6} \mathrm{~km}^{2}$. This area has the most continuous soil in both the horizontal and vertical planes, the most typical loess geomorphic landform and eroded terrain, including the Yuan (large flat surface with little or no erosion), Liang (long narrow range of hills), Mao (oval or round loess hill), and various gullies (Yang et al., 1988; Jia et al., 2015; Zhao et al., 2017b). Annual mean temperature is $14.3^{\circ} \mathrm{C}$ in the southeast and $3.6^{\circ} \mathrm{C}$ in the northwest. Mean annual precipitation increases from 150 (northwest) to $800 \mathrm{~mm}$ (southeast) along the south-north transect, with $55 \%-78 \%$ of the annual precipitation falling in June to September. Mean annual evaporation is $1400-2000 \mathrm{~mm}$ (He et al., 2003). The soil is weakly cohesive, with high infiltration and erosion rates. Soil fertility loss and land degradation are common phenomena in the area due to severe soil and water loss (Zhao et al., 2016).

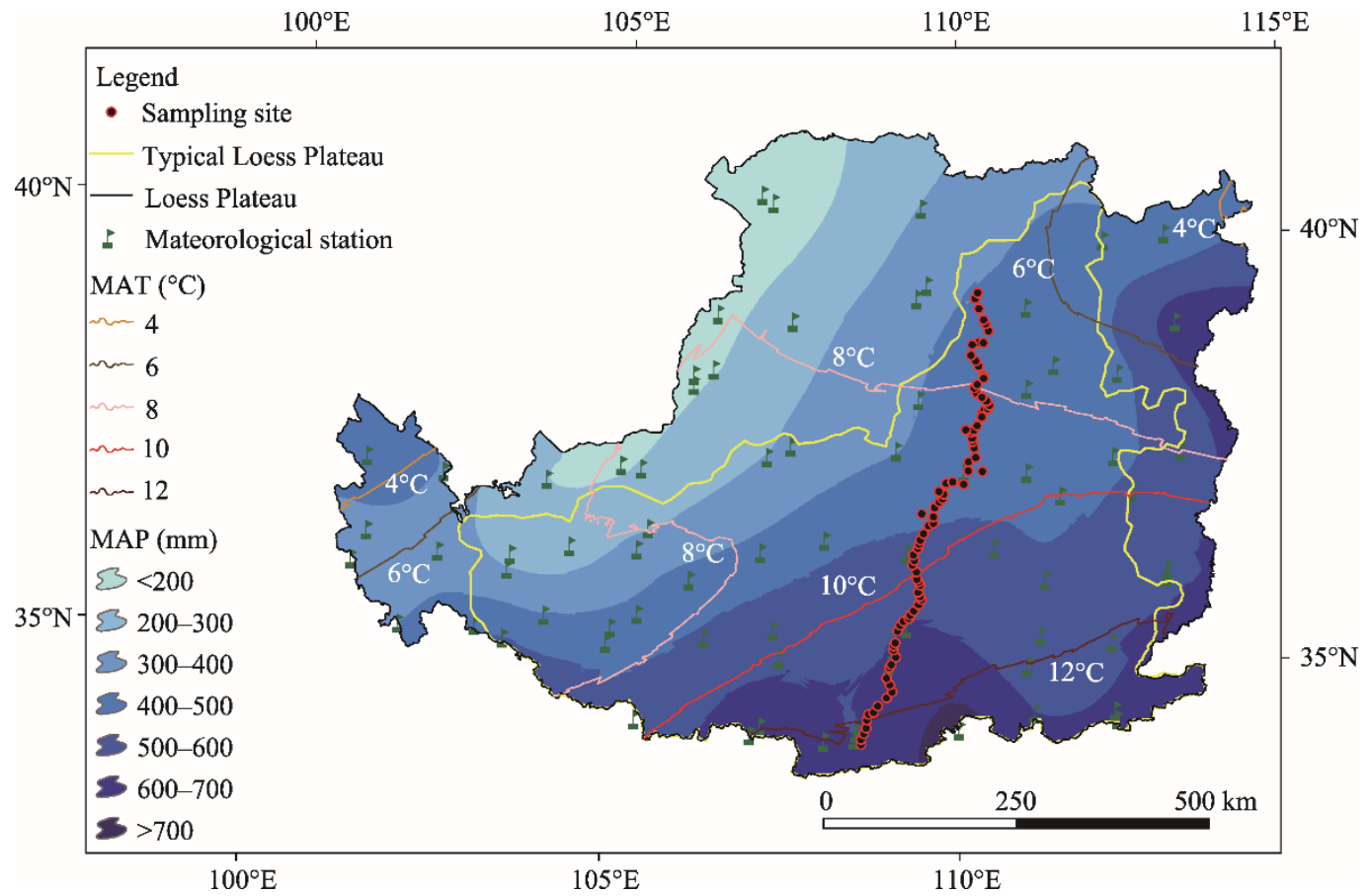

Fig. 1 Location of the 86 sampling sites and distributions of mean annual temperature (MAT) and mean annual precipitation (MAP) along the south-north transect across the Loess Plateau of China

\subsection{Soil sampling and data collection}

An approximately 860-km long south-north transect across the typical Loess Plateau was set up, as shown in Figure 1. A total of 86 sampling sites were selected at an approximate interval of $10 \mathrm{~km}$ along the south-north transect. It should be noted that all the sampling sites were located away from gullies and bench lands where the groundwater table is likely to be relatively shallow to avoid any effect of groundwater on SWC (including capillary rise into the root zone), and located at least 200 $\mathrm{m}$ away from the road to reduce the effect of road on SWC. Each sampling site was purposively selected to adequately represent the main land use, topography, soil type and vegetation type within the range of vision.

An aluminum neutron probe access tube $(5.2 \mathrm{~m}$ in length) was installed at each sampling site. Slow neutron counts were taken at an interval of $0.2 \mathrm{~m}$ in the $0.0-5.0 \mathrm{~m}$ soil profile using the CNC503DR Hydro-Probe (Beijing Super Power Company, Beijing, China). We calculated the volumetric SWC $(\theta ; \%)$ using the slow neutron count rate $(\mathrm{CR})$ based on the calibration curve as follows:

$$
\theta=0.5891 \times \mathrm{CR}+0.0089 \text {. }
$$

This calibration curve was obtained from the SWC data for the same area and was considered valid for the $0.0-5.0 \mathrm{~m}$ soil depth $\left(R^{2}=0.93, P<0.001\right)$. At each sampling site, $\mathrm{SWC}$ was calculated 
for 25 soil depths (0.2 $\mathrm{m}$ for each) in the growing seasons from June 2013 to September 2016 (including June, August, September, October and November in 2013; April, June, July, August and September in 2014; April, May, June, July and September in 2015; and May, July and November in 2016) and each measurement process was completed within 3-4 d. The coordinates and altitude of each sampling site were obtained using a GPS receiver ( $5 \mathrm{~m}$ resolution).

At each sampling site, disturbed soil samples were taken from 11 soil layers $(0.0-0.1,0.1-0.2$, $0.2-0.4,0.4-0.6,0.6-0.8,0.8-1.0,1.0-1.5,1.5-2.0,2.0-3.0,3.0-4.0$ and $4.0-5.0 \mathrm{~m}$ ) in the $0.0-5.0$ $\mathrm{m}$ soil profile using a soil auger $(5 \mathrm{~cm}$ in diameter). Thus, a total of 946 disturbed soil samples (86 sampling sites $\times 11$ soil layers) were collected for laboratory analyses. The disturbed soil samples were air-dried and passed through $2.00-\mathrm{mm}$ mesh sieves to measure clay content (\%; USDA soil taxonomy) by the laser diffraction grain size analyser (Mastersizer 2000, Malvern Instruments, England), and through $0.25-\mathrm{mm}$ mesh sieves to measure soil organic carbon (SOC; $\mathrm{g} / \mathrm{kg}$ ) using dichromate oxidation method.

Slope gradient $\left(^{\circ}\right)$ was measured using a geological compass. To determine the effect of land use on SWC, we labeled cropland, forestland and grassland as 0,1 and 2, respectively, in decreasing order of mean SWC. Climate data were obtained from 73 meteorological stations of the China Climatic Data Sharing Service System (http:/cdc.cma.gov.cn/) for the Loess Plateau region (Fig. 1). The dataset consists mean annual precipitation (MAP; mm), mean annual evaporation (MAE; $\mathrm{mm}$ ), mean annual temperature (MAT; ${ }^{\circ} \mathrm{C}$ ) and mean annual dryness (MAD) index for the period 1953-2013. Based on station-specific data, we developed a continuous data surface of climatic factors via kriging interpolation at $100 \mathrm{~m} \times 100 \mathrm{~m}$ resolution. Then, we extracted the specific climatic factors for each sampling site by resampling in ArcGIS software (Version: ESRI ${ }^{\circledR}$ $\operatorname{ArcMap}^{\mathrm{TM}}$ 10.2). The variations of land use, MAE, altitude and MAD along the south-north transect are shown in Figure 2.

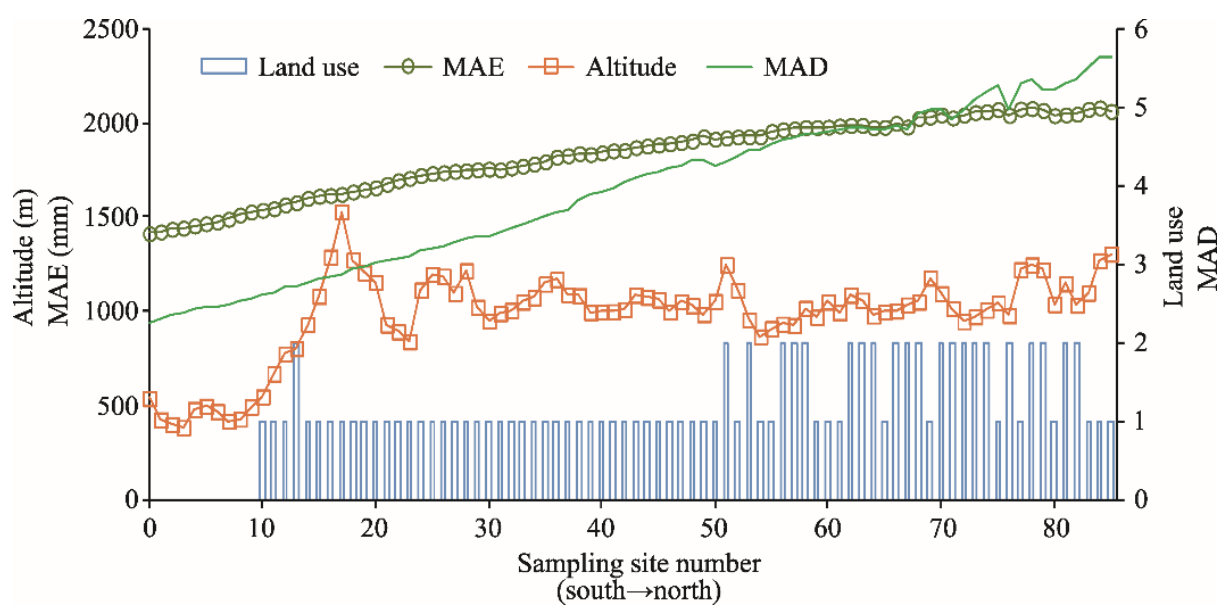

Fig. 2 Variations of mean annual evaporation (MAE), mean annual dryness (MAD) index, altitude and land use along the south-north transect across the typical Loess Plateau. Note that 0,1 and 2 in the vertical axis for the land use indicate cropland, forestland and grassland, respectively.

\subsection{Data analyses}

In this study, coefficient of variation (CV) was used to evaluate the temporal stability of SWC at the site scale. Specifically, CV is defined as the variability of SWC in a time domain and is expressed as:

$$
\mathrm{CV}=\frac{\sigma\left(\theta_{i}\right)}{\bar{\theta}_{i}} \times 100 \%,
$$

where $\sigma\left(\theta_{i}\right)$ and $\bar{\theta}_{i}$ are the standard deviation and mean of SWC over time for the $i^{\text {th }}$ sampling site, respectively (Starr, 2005; Hu et al., 2012). The degree of variation is usually divided into three 
levels: weak variability when $\mathrm{CV} \leq 10 \%$, moderate variability when $10 \%<\mathrm{CV} \leq 100 \%$ and strong variability when $\mathrm{CV}>100 \%$ (Jia and Shao, 2014). In this study, the WAL was identified as the CV of SWC $>10 \%$.

Based on the current understanding and definition of WAL, we developed three quantitative indices of WAL: (1) WAL thickness (WAL-T; m); (2) mean coefficient of variation in SWC within WAL (WAL-CV; \%); and (3) mean SWC within WAL (WAL-SWC; \%). Figure 3 is a schematic representation of the three quantitative indices of WAL in the soil profile at a sampling site with MAP of 500-600 $\mathrm{mm}$ in forestland.

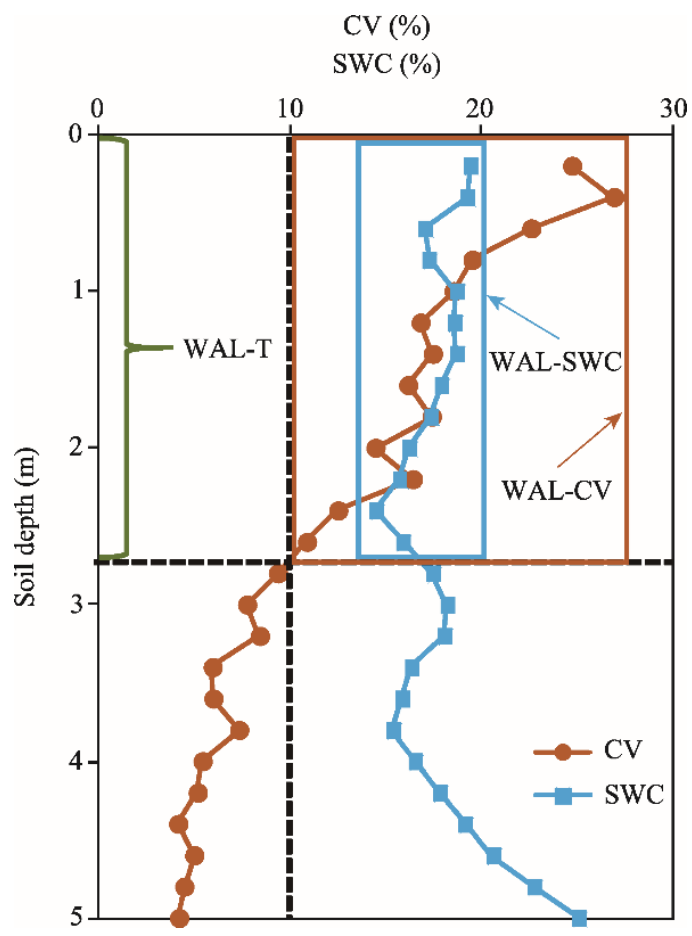

Fig. 3 Schematic representation of the three quantitative indices of water active layer (WAL) in the soil profile at a sampling site with MAP of 500-600 mm in forestland. SWC, soil water content (\%); CV, coefficient of variation (\%); WAL-T, soil water active layer thickness (m); WAL-SWC, mean soil water content within water active layer (\%); WAL-CV, mean coefficient of variation in soil water content within water active layer (\%). The mean values of SWC and CV inside of the corresponding frames were WAL-SWC and WAL-CV, respectively.

To illustrate the basic patterns of CV, SWC, clay content and SOC along the south-north transect, we developed maps of the respective distributions using universal kriging interpolation in ArcGIS software (ESRI ${ }^{\circledR}$ ArcMap $\left.{ }^{\text {TM }} 10.2\right)$. We removed the spatial trends of the first or second order in the dataset before interpolation (Zhao et al., 2016). Furthermore, we standardized the data (with zero mean and unit variance) to eliminate the effect of index dimension and quantity of data before data analysis (Xiong et al., 2016). Redundancy analysis (RDA) was done to determine the possible relationships among WAL indices and measured environmental factors. The nine environmental factors were divided into two groups as climatic (MAP, MAE, MAT and MAD) and local (altitude, slope, land use, clay content and SOC) factors. Furthermore, partial redundancy analysis (PRDA) was used to measure the relative roles of these climatic and local factors affecting the WAL. Both RDA and PRDA were conducted using the "Vegan" package (http://cran.rproject.org/web/packages/vegan) in R statistics version 3.3.2.

\subsection{Pedotransfer function (PTF) development}

For each sampling site, four climatic and five local factors were used to estimate the indices of WAL (WAL-T, WAL-CV and WAL-SWC). Finally, 86 datasets were used to develop PTFs by both multiple linear regression (MLR) and artificial neural network (ANN) methods. 
MLR is a traditional PTF development method that is widely used in soil studies (Motaghian and Mohammadi, 2011; Patil and Chaturvedi, 2012). ANN can be used to describe the systems without assumed model structure (Motaghian and Mohammadi, 2011). ANN approach is used for modeling the relationships between soil parameters and other measurable PTF development properties (Minasny and Mcbratney, 2002; Xiong et al., 2011). In this study, a trial and error method was used to determine the specific numbers of hidden layers and neurons in each layer of the ANN (Xiong et al., 2011). A pure linear function and sigmoid activation function were selected for the output layer and hidden layer, respectively.

The 86 datasets were randomly divided into two subsets: subset- 1 contained 58 PTF developing records $(70 \%$ of the total dataset) and subset- 2 contained 28 validation records $(30 \%$ of the total dataset). Coefficient of determination $\left(R^{2}\right)$ and root mean square error (RMSE) were used to evaluate the accuracy of the PTFs. The calculations of $R^{2}$ and RMSE can be found in Wang et al. (2014).

\section{Results and discussion}

\subsection{Spatial patterns of CV, SWC and WAL}

The vertical distribution of CV of SWC showed a strong depth dependency for the 0.0-5.0 m soil profile along the south-north transect (Fig. 4a). The CV was maximum in the surface soil layer, and the value decreased with increasing soil depth. The distribution of SWC showed an obvious spatial trend. SWC was relatively small and uniformly distributed in the $0.0-5.0 \mathrm{~m}$ soil profile in the middle region of the south-north transect (Fig. 4b). Furthermore, SWC was relatively high in the southern and northern regions of the south-north transect, and generally increased with increasing soil depth.

The temporal variation in SWC (described as CV) was generally greater in the upper soil layers than in the deep soil layers (Fig. 4a). This was because SWC in the upper soil layers was more sensitive to precipitation, evaporation, vegetation uptake and human activity. For example, surface soil layer absorbs most of the infiltrating water and is therefore the main source of water loss via evapotranspiration. The fluctuation of SWC in the deep soil layers was mitigated by the insulating effect of the upper soil layers. This causes a stronger temporal stability of SWC in the deep soil layers than in the upper soil layers (Choi and Jacobs, 2007; Hu et al., 2010).

However, the trend was exceptional for sampling sites 49-51, where the CV of SWC was higher in the 3.0-4.5 m soil layers than in the other layers (Fig. 4a). The huge plant water uptake from the deep soil layers was responsible for this exceptional case (Zhao et al., 2017a). Wang et al. (2016) reported that in the Yellow River Basin of the Loess Plateau, there was an obvious decline in sediment concentration and runoff due to massive afforestation in the region. However, the conversion of cropland into forestland has induced soil desiccation in the deep soil layers across the plateau (Jia et al., 2017). Wang et al. $(2011,2014)$ noted that the depth of depleted soil water was sometimes over $5.0 \mathrm{~m}$. This is due mainly to the widespread introduction of tree plants with deep root systems (e.g., Caragana korshinskii, Robinia pseudoacacia and Pinus tabulaeformis). The extent of dry soil layer in forestland is more severe than that in cropland or grassland. The higher SWC variation in the 3.0-4.5 m soil layer among sites 49-51 was attributed to the huge plant water uptake and was not the result of combined effect of climate and vegetation. Thus, this soil layer (3.0-4.5 m soil layer among sites 49-51) cannot considered to be a WAL in the study area.

As shown in Figure 5, there was a quadric trend in WAL-T, WAL-CV and WAL-SWC from the south to the north of the transect. The values of WAL-T and WAL-CV were greater for sampling sites in the middle region than in the southern and northern regions of the south-north transect. However, for WAL-SWC curve, the dip was maximum in the middle region of the south-north transect (Fig. 5). Based on the quadratic regression functions (not shown), the peak of WAL-T and WAL-CV and the dip of WAL-SWC were in the regions around sites 55, 47 and 67 . The high gradient and thick distribution of WAL could be due to the low clay content, deep root distribution 
and high precipitation in the region between sites 47 and 67. Soil water in the middle region of the south-north transect was more active, with WAL-T exceeding $4.0 \mathrm{~m}$ at some sampling sites. Average WAL-T in the study area was $2.0 \mathrm{~m}$.

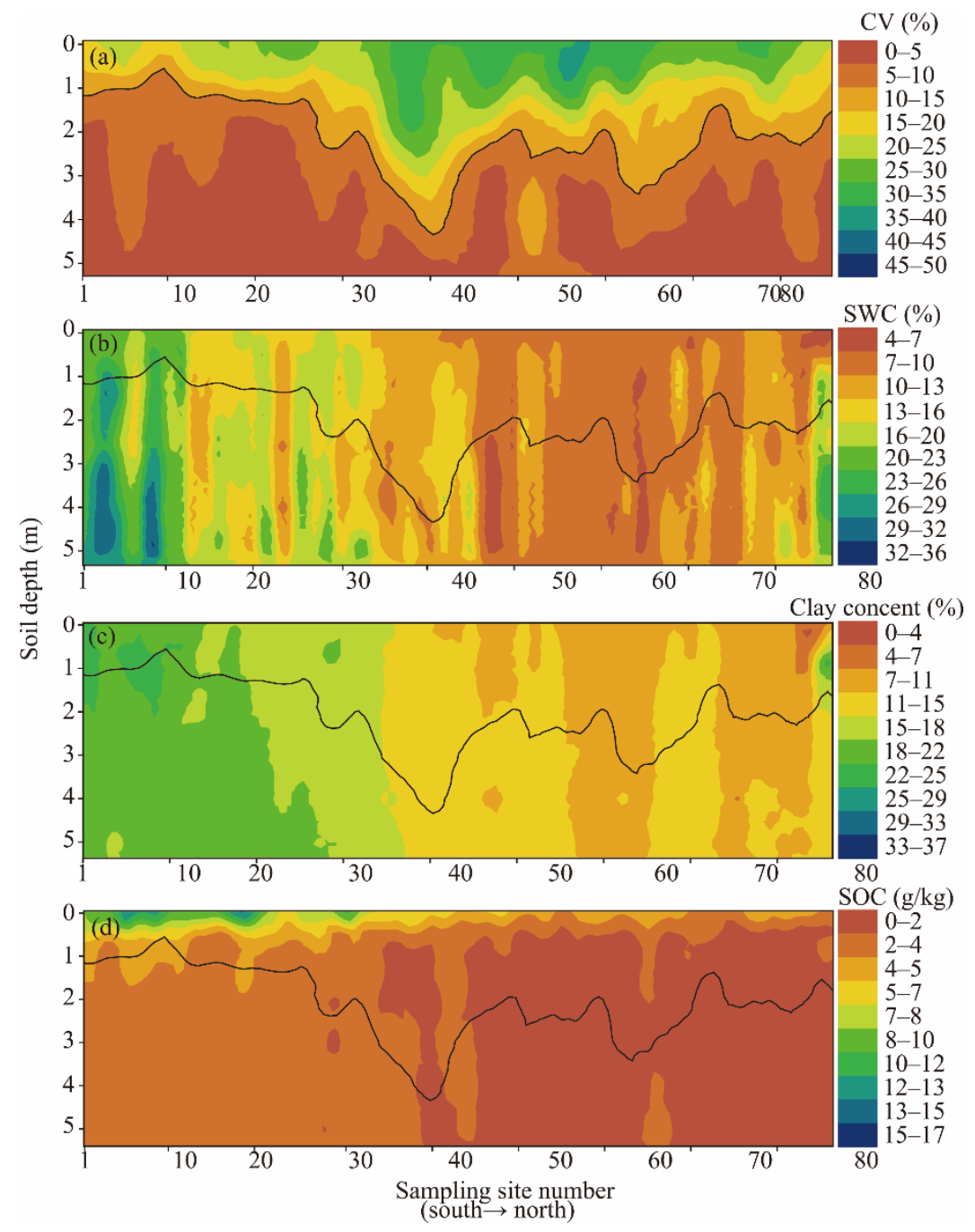

Fig. 4 Profile distributions of CV (a), SWC (b), clay content (c) and SOC (d) along the south-north transect across the typical Loess Plateau. Note that the black curve is the boundary line of $\mathrm{CV}>10 \%$.

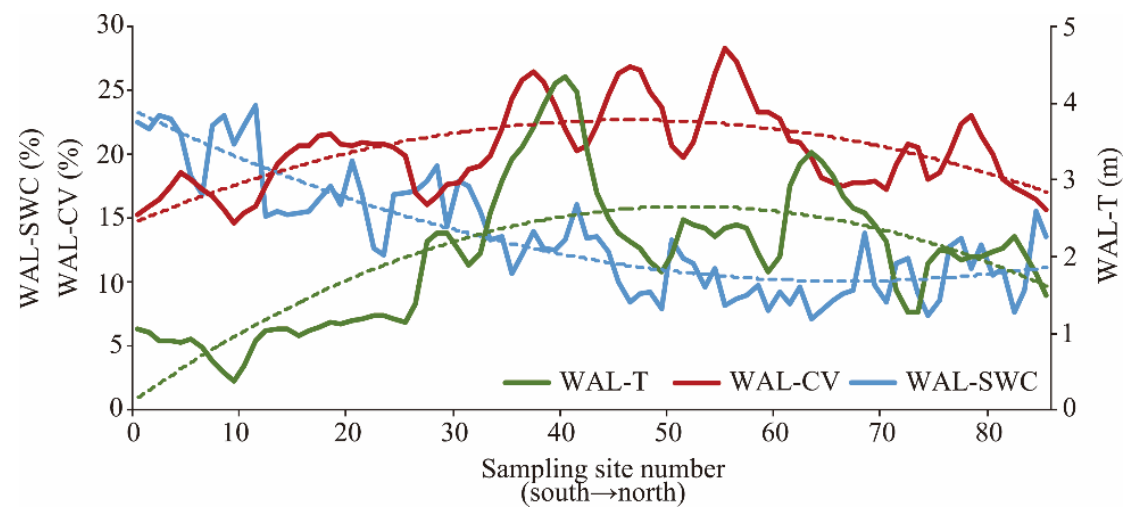

Fig. 5 Distributions of WAL-T, WAL-SWC and WAL-CV along the south-north transect across the typical Loess Plateau. Note that the dotted lines are the trends in WAL-T, WAL-SWC and WAL-CV. 


\subsection{Factors driving WAL}

In the RDA, the ordination biplot was used to describe the relationships between WAL indices and related environmental factors (Fig. 6). WAL-T was positively correlated with MAE, MAD, altitude and land use, but negatively correlated with SOC, clay content, MAP and MAT. However, the correlations of WAL-SWC with environmental factors were opposite to those of WAL-T. Specifically, WAL-SWC was positively correlated with SOC, clay content, MAP and MAT, and negatively correlated with MAE, MAD, altitude and land use. Furthermore, WAL-CV was weakly associated with all these factors, except slope gradient.

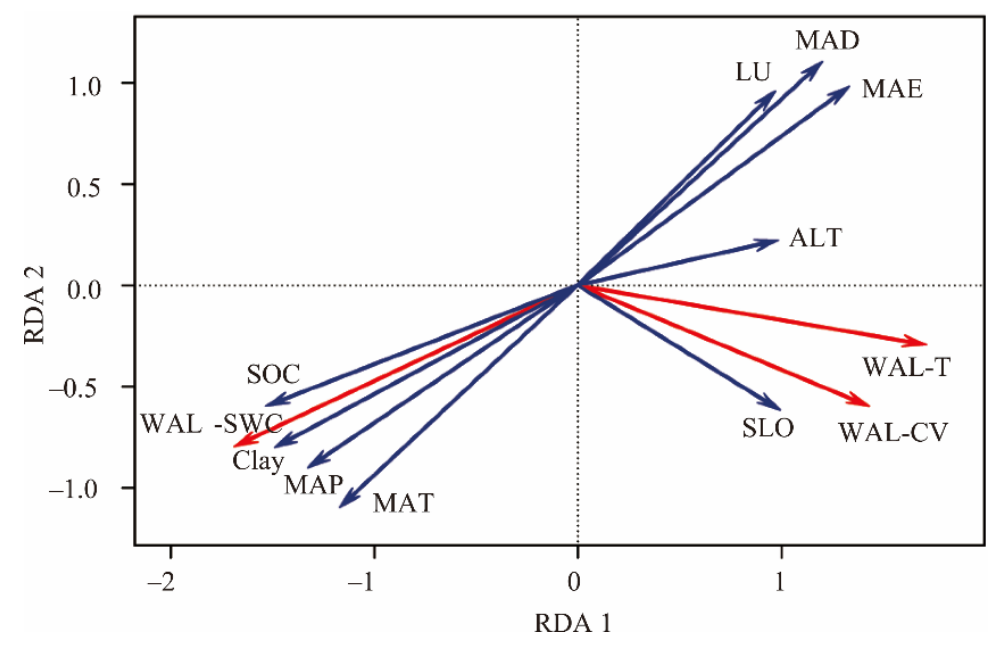

Fig. 6 Relationships between WAL indices (WAL-T, WAL-SWC and WAL-CV) and related environmental factors along the south-north transect across the typical Loess Plateau. MAP, mean annual precipitation; MAE, mean annual evaporation; MAT, mean annual temperature; MAD, mean annual dryness; ALT, altitude; SLO, slope gradient; LU, land use; Clay, clay content; SOC, soil organic carbon.

The high values of clay content and SOC content enhance the soil water holding capacity (Zhao et al., 2017b). In the study, the trends in clay content and SOC content decreased along the transect from the south to the north. This resulted in a greater soil water holding capacity in the southern region of the transect than in the northern region. With increasing precipitation and decreasing evaporation from the north to the south, the southern region of the transect had a higher WAL-SWC than the northern region. In the Loess Plateau, regions with high precipitation are generally accompanied by higher contents of clay and SOC (Figs. 1 and 4c and d). Although precipitation in the southern region of the transect had favorable conditions for soil water recharge in the deep soil layers, the low soil water infiltration as a result of high contents of clay and SOC limited the depth of rainfall infiltration (Wang et al., 2012). Furthermore, cropland was the dominant land use type in the southern region of the transect. Cropland uses less deep soil water than forestland and grassland. As a result, regions with high precipitation did not show a thick WAL-T or high WAL$\mathrm{CV}$ in the study area.

As shown in Table 1, all the nine environmental factors were critical at $P<0.001$ level in the determination of the indices and spatial patterns of WAL. The relative importance of each environmental factor was also examined when they were treated separately as shown in Table 1. All the climatic factors (MAP, MAE, MAT and MAD) strongly influenced the indices of WAL $\left(R^{2}>0.67, P<0.001\right)$. Clay content $\left(R^{2}=0.81, P<0.001\right)$ and SOC $\left(R^{2}=0.78, P<0.001\right)$ were the highest two local contributors and the most important environmental factors driving WAL characteristics. This is because both clay and SOC affect soil water infiltration and water holding capacity (Jia et al., 2015; Zhao et al., 2017b), which directly determine the range and degree of variation in WAL. 
Table 1 Results of redundancy analysis (RDA) for the nine environmental factors in the determination of the indices and spatial patterns of WAL (water active layer)

\begin{tabular}{lccc}
\hline & Environmental factors & $R^{2}$ & $P$ \\
\hline Climatic factors & MAP & 0.71 & $<0.001$ \\
& MAE & 0.74 & $<0.001$ \\
& MAT & 0.67 & $<0.001$ \\
Local factors & MAD & 0.69 & $<0.001$ \\
& ALT & 0.30 & $<0.001$ \\
& SLO & 0.34 & $<0.001$ \\
& LU & 0.46 & $<0.001$ \\
& Clay & 0.81 & $<0.001$ \\
\hline
\end{tabular}

Note: MAP, mean annual precipitation; MAE, mean annual evaporation; MAT, mean annual temperature; MAD, mean annual dryness; ALT, altitude; SLO, slope gradient; LU, land use; Clay, clay content; SOC, soil organic carbon.

PRDA was further used to distinguish the relative importance of climatic and local factors affecting WAL indices. The results showed that the nine environmental factors explained $69.3 \%$ of the total variability of WAL, which also contributed greatly to WAL indices (Fig. 7). Specifically, the measured five local factors explained only $4.9 \%$ of the total variability of WAL indices, while the four climatic factors explained $10.1 \%$ of the total variability of WAL indices. Furthermore, the interaction between climatic factors and local factors accounted for $54.3 \%$ of the total variability of WAL indices. This further indicated that both climatic and local factors were largely responsible for the spatial patterns and indices of WAL. However, compared with local factors, climatic factors influenced the spatial patterns and indices of WAL more importantly.

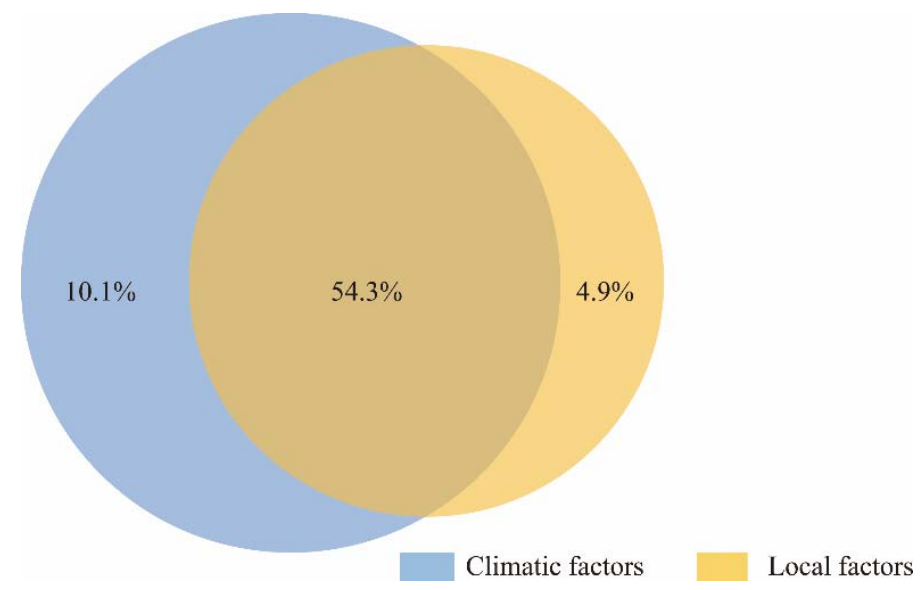

Fig. 7 Relative contributions of climatic and local factors to the total variability of WAL indices

\subsection{Estimation of WAL indices}

In this study, both MLR and ANN analyses were used to estimate WAL indices (WAL-T, WALSWC and WAL-CV), realted to four climatic factors (MAP, MAE, MAT and MAD) and five local factors (altitude, slope gradient, land use, clay content and SOC). We conducted a stepwise analysis for the different combinations of the nine environmental factors, and selected the six best performing PTFs developed by MLR and ANN, which are listed in Table 2. PTFs developed by ANN respectively explained $86 \%, 81 \%$ and $64 \%$ of the total variations in WAL-T, WAL-SWC and WAL-CV. The PTF with three climatic (MAD, MAP and MAT) and one local (SOC) factors had the best performance regarding WAL-T estimation. PTF of clay content and SOC was the best input combination for estimating WAL-SWC, and PTF of slope gradient, clay content, MAT and SOC was the best input combination for estimating WAL-CV. 
Table 2 Coefficient of determination $\left(R^{2}\right)$ and root mean square error (RMSE) of estimated WAL-T, WAL-SWC and WAL-CV using multiple linear regression (MLR) and artificial neural network (ANN) methods

\begin{tabular}{ccccc}
\hline Method & Index & PTF combination & $R^{2}$ & RMSE \\
\hline \multirow{2}{*}{ MLR } & WAL-T & SOC+MAD+MAP+MAT & 0.67 & 0.88 \\
& WAL-SWC & Clay+SOC & 0.78 & 2.17 \\
& WAL-CV & SLO+Clay+MAT+SOC & 0.49 & 2.31 \\
& WAL-T & SOC+MAD+MAP+MAT & 0.86 & 0.36 \\
ANN & WAL-SWC & Clay+SOC & 0.81 & 2.05 \\
& WAL-CV & SLO+Clay+MAT+SOC & 0.64 & 2.15 \\
\hline
\end{tabular}

Note: WAL-T, water active layer thickness; WAL-SWC, mean soil water content within WAL;WAL-CV, mean coefficient of variation in soil water content within WAL; PTF, pedotransfer function.

As shown in Table 2 and Figure 8, the accuracy of PTF-estimated WAL indices by ANN was significantly better than that by MLR. For the estimation of WAL-T, ANN-built PTF performed well $\left(R^{2}=0.86\right.$, RMSE $=0.36$; Table 2$)$ and the trend line was almost an overlay of the $1: 1$ line (Fig. 8a). By contrast, MLR-built PTF overestimated WAL-T and the accuracy was significantly lower than that of ANN-built PTF (Fig. 8a). The PTF-estimated WAL-SWC by MLR and ANN methods was similar when WAL-SWC value is higher than $13 \%$ (Fig. $8 \mathrm{~b}$ ). However, when WAL-SWC value is lower than $13 \%$, MLR-built PTF underestimated WAL-SWC, showing a smaller $R^{2}$ value compared with ANN-built PTF $\left(R^{2}=0.78\right.$ and $R^{2}=0.81$, respectively; Table 2$)$. It should be noted that both ANN- and MLR-built PTF failed to accurately describe the variations in WAL-CV very well (Table 2). However, the performance of ANN-built PTF was still significantly better than that of MLR-built PTF (Fig. 8c).
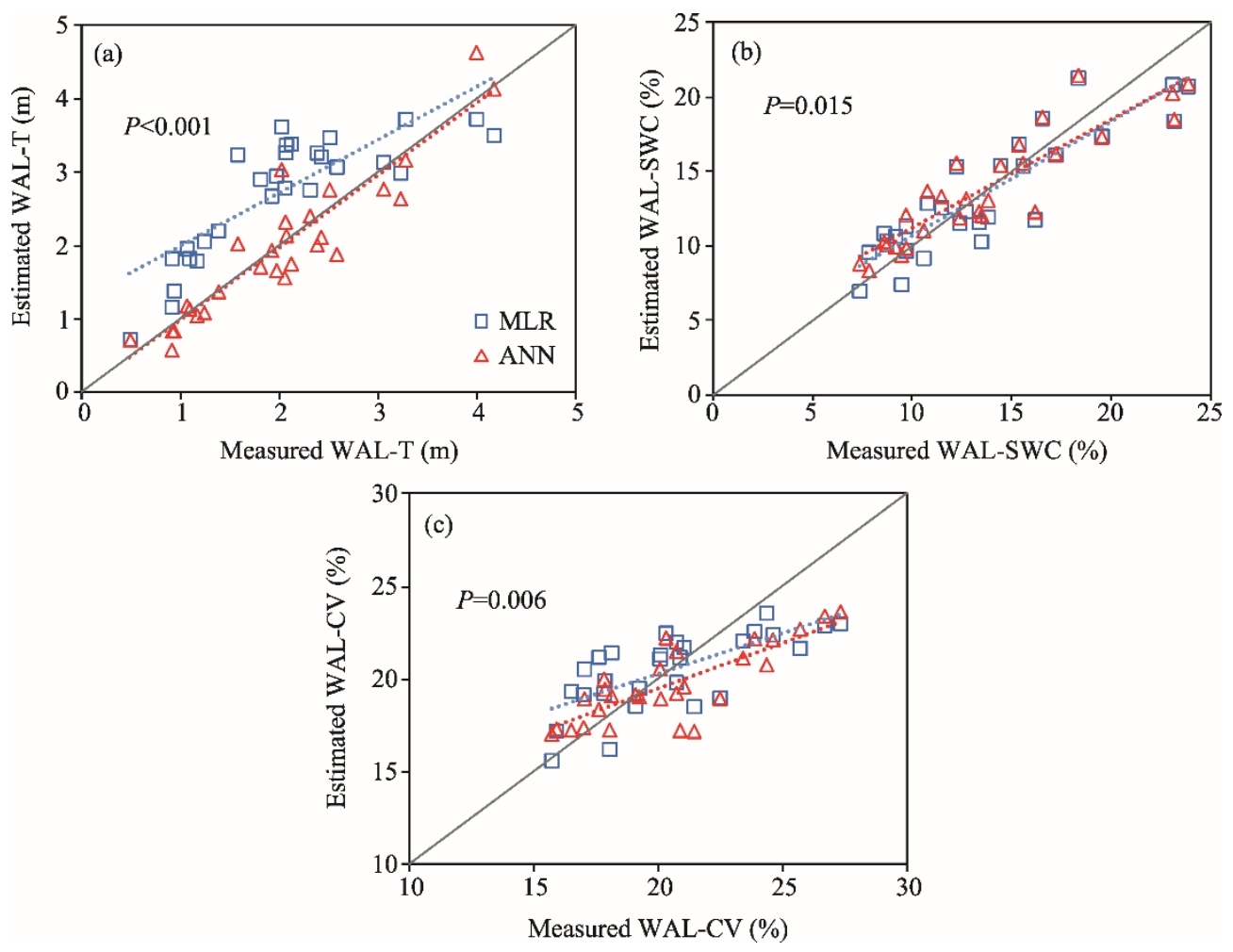

Fig. 8 Comparisons of estimated WAL-T (a), WAL-SWC (b) and WAL-CV (c) using multiple linear regression (MLR) and artificial neural network (ANN) methods. Note that the blue and red dotted lines are the trend lines for indices estimations using MLR and ANN, respectively; the gray line indicates the 1:1 line; and $P$ is the difference of estimations between MLR and ANN methods by $T$-test ( $95 \%$ significance level). 
This finding was consistent with the result of ANN reported in other studies (e.g., Motaghian and Mohammadi, 2011; Patil and Chaturvedi, 2012). Patil and Chaturvedi (2012) found that the overall performance of ANN-built PTF was better than that of MLR-built PTF. Compared with MLR, ANN had a high capability for modeling complex problems. ANN can overcome the difficulties associated with the use of physically-based methods for simulating complex relationships. It can better relate the inputs and outputs of a system even when the physical conditions are very complex (Xiong et al., 2011). ANN could acquire experimental knowledge to balance weights through training, thus making it very tolerant and robust. In this study, ANN-built PTF performed significantly better than MLR-built PTF and was therefore recommended for use in estimating WAL indices in the Loess Plateau. Nevertheless, MLR-built PTF was still useful for regions with no adequate data for ANN analysis.

\subsection{Implications for vegetation restoration}

WAL is a good indicator for evaluating the plant-available soil water-holding capacity. The activity of WAL (quantified by WAL-CV) reflects the frequency of water exchange between soil-plant and soil-atmosphere continuums. SWC of WAL (WAL-SWC) reflects soil water holding capacity and soil water output capability of the soil water reservoir. The thickness of WAL (WAL-T) shows the range of the soil layer with active soil water output and precipitation recharge. In the Loess Plateau, knowledge of WAL is critical for developing vegetation restoration strategies, particularly for the choice of planting zones, vegetation types and management measures.

In the middle region of the south-north transect where WAL-T is thick, deep soil water depletion could be replenished under natural conditions and deep-rooted plants are suitable for cultivation in this region. However, given the low WAL-SWC and small soil water carrying capacity of vegetation, attention should be refocused on vegetation density when designing vegetation restoration strategies in the region. Soils in the southern region of the south-north transect have a relatively high WAL-SWC, which can support the growth of high water-consuming plants. However, in the southern region, WAL-T is thicker than that in the middle region and deep soil water recharge is limited, thus the cultivation of deep-rooted plants in the region is not recommended. Compared with the middle region of the south-north transect, WAL-SWC in the northern region is higher. Irrespective of the smaller WAL-T and WAL-CV, grassland ecosystem is still the best choice for re-vegetation in this region because of its low soil water use.

\section{Conclusions}

In this study, we investigated the spatial characteristics of WAL and the driving factors in the 0.0 $5.0 \mathrm{~m}$ soil profile along a south-north transect across the typical Loess Plateau. The distributions of WAL-T, WAL-CV and WAL-SWC followed a quadric function along the south-north transect across the plateau. Soil water in the middle region of the south-north transect had a stronger activity due to relatively high precipitation and low soil clay content. In the middle region, soil water exchange occurred deeper than $2.0 \mathrm{~m}$ and WAL-T even exceeded $4.0 \mathrm{~m}$ at some sampling sites. In other regions, however, soil water exchange was averagely within the $2.0 \mathrm{~m}$ soil layers. The characteristics of WAL were the result of the combined effects of climatic and local factors, with the role of climatic factors being stronger ( $10.1 \%$ for climatic factors versus $4.9 \%$ for local factors). The analysis suggested that climatic conditions are the critical consideration in developing vegetation restoration strategies in the Loess Plateau. For WAL-T, WAL-CV and WAL-SWC, PTFs built from ANN approach significantly performed better than those from MLR approach, and therefore, ANN approach was recommended for the estimation of WAL indices in the Loess Plateau.

\section{Acknowledgements}

This study was supported by the National Natural Science Foundation of China $(41530854,41571130081)$, the National Key Project for Research and Development (2016YFC0501605) and the Youth Innovation Promotion Association of Chinese Academy of Sciences (2017076). We thank the anonymous reviewers and editors for their valuable comments and suggestions for improving the manuscript. 


\section{References}

Biswas A, Si B C. 2011. Application of continuous wavelet transform in examining soil spatial variation: A review. Mathematical Geosciences, 43(3): 379-396.

Brocca L, Melone F, Moramarco T, et al. 2010. Spatial-temporal variability of soil moisture and its estimation across scales. Water Resources Research, 46(2): W02516.

Chen H, Shao M A, Li Y Y. 2008. Soil desiccation in the Loess Plateau of China. Geoderma, 143(1-2): 91-100.

Choi M, Jacobs J M. 2007. Soil moisture variability of root zone profiles within SMEX02 remote sensing footprints. Advances in Water Resources, 30(4): 883-896.

Crave A, Gascuel-Odoux C. 1997. The influence of topography on time and space distribution of soil surface water content. Hydrological Processes, 11(2): 203-210.

Gao L, Shao M A, Peng X H, et al. 2015. Spatio-temporal variability and temporal stability of water contents distributed within soil profiles at a hillslope scale. CATENA, 132: 29-36.

Gasch C K, Brown D J, Campbell C S, et al. 2017. A field-sensor network data set for monitoring and modeling the spatial and temporal variation of soil water content in a dryland agricultural field. Water Resources Research, 53(12): 10878-10887.

He X B, Li Z B, Hao M D, et al. 2003. Down-scale analysis for water scarcity in response to soil-water conservation on Loess Plateau of China. Agriculture, Ecosystems \& Environment, 94(3): 355-361.

Heathman G C, Larose M, Cosh M H, et al. 2009. Surface and profile soil moisture spatio-temporal analysis during an excessive rainfall period in the Southern Great Plains, USA. CATENA, 78(2): 159-169.

Hu W, Shao M, Han F P, et al. 2010. Watershed scale temporal stability of soil water content. Geoderma, 158(3-4): 181-198.

Hu W, Tallon L K, Si B C. 2012. Evaluation of time stability indices for soil water storage upscaling. Journal of Hydrology, 475 : 229-241.

Hupet F, Vanclooster M. 2002. Intraseasonal dynamics of soil moisture variability within a small agricultural maize cropped field. Journal of Hydrology, 261(1-4): 86-101.

Jia X X, Shao M A, Wei X R, et al. 2013. Hillslope scale temporal stability of soil water storage in diverse soil layers. Journal of Hydrology, 498: 254-264.

Jia X X, Shao M A, Zhang C, et al. 2015. Regional temporal persistence of dried soil layer along south-north transect of the Loess Plateau, China. Journal of Hydrology, 528: 152-160.

Jia X X, Shao M A, Zhu Y J, et al. 2017. Soil moisture decline due to afforestation across the Loess Plateau, China. Journal of Hydrology, 546: 113-122.

Jia X X, Shao M A, Yu D X, et al. 2019. Spatial variations in soil-water carrying capacity of three typical revegetation species on the Loess Plateau, China. Agriculture, Ecosystems \& Environment, 273: 25-35.

Jia Y H, Shao M A. 2014. Dynamics of deep soil moisture in response to vegetational restoration on the Loess Plateau of China. Journal of Hydrology, 519: 523-531.

Li Y S. 1983. The properties of water cycle in soil and their effect on water cycle for land in the Loess Plateau. Acta Ecologica Sinica, 3: 91-101. (in Chinese)

Minasny B, Mcbratney A B. 2002. Uncertainty analysis for pedotransfer functions. European Journal of Soil Science, 53(3): 417429.

Mohanty B P, Famiglietti J S, Skaggs T H. 2000. Evolution of soil moisture spatial structure in a mixed vegetation pixel during the Southern Great Plains 1997 (SGP97) Hydrology Experiment. Water Resources Research, 36(12): 3675-3686.

Motaghian H R, Mohammadi J. 2011. Spatial estimation of saturated hydraulic conductivity from terrain attributes using regression, kriging, and artificial neural networks. Pedosphere, 21(2): 170-177.

Nyberg L. 1996. Spatial variability of soil water content in the covered catchment at Gårdsjön, Sweden. Hydrological Processes, 10(1): 89-103.

Patil N G, Chaturvedi A. 2012. Estimation of bulk density of waterlogged soils from basic properties. Archives of Agronomy and Soil Science, 58(5): 499-509.

Starr G C. 2005. Assessing temporal stability and spatial variability of soil water patterns with implications for precision water management. Agricultural Water Management, 72(3): 223-243.

Wang J, Ge Y, Heuvelink G B M, et al. 2015. Upscaling in situ soil moisture observations to pixel averages with spatio-temporal geostatistics. Remote Sensing, 7(9): 11372-11388.

Wang S, Fu B J, Piao S L, et al. 2016. Reduced sediment transport in the Yellow River due to anthropogenic changes. Nature Geoscience, 9: 38-41. 
Wang X C, Li J, Tahir M N, et al. 2012. Validation of the EPIC model and its utilization to research the sustainable recovery of soil desiccation after alfalfa (Medicago sativa L.) by grain crop rotation system in the semi-humid region of the Loess Plateau. Agriculture, Ecosystems \& Environment, 161: 152-160.

Wang Y Q, Shao M A, Zhu Y J, et al. 2011. Impacts of land use and plant characteristics on dried soil layers in different climatic regions on the Loess Plateau of China. Agricultural and Forest Meteorology, 151(4): 437-448.

Wang Y Q, Shao M A, Liu Z P. 2012. Pedotransfer functions for predicting soil hydraulic properties of the Chinese Loess Plateau. Soil Science, 177(7): 424-432.

Wang Y Q, Shao M A, Liu Z P, et al. 2014. Prediction of bulk density of soils in the Loess Plateau region of China. Surveys in Geophysics, 35(2): 395-413.

Xiong W, Li J, Chen Y Y, et al. 2016. Determinants of community structure of zooplankton in heavily polluted river ecosystems. Scientific Reports, 6: 22043.

Xiong Y W, Wallach R, Furman A. 2011. Modeling multidimensional flow in wettable and water-repellent soils using artificial neural networks. Journal of Hydrology, 410(1-2): 92-104.

Yang Q Y, Zhang B P, Zheng D. 1988. On the boundary of the Loess Plateau. Journal of Natural Resources, 3(1): 9-15. (in Chinese)

Zhao C L, Shao M A, Jia X X, et al. 2016. Particle size distribution of soils (0-500 cm) in the Loess Plateau, China. Geoderma Regional, 7(3): 251-258.

Zhao C L, Jia X X, Zhu Y J, et al. 2017a. Long-term temporal variations of soil water content under different vegetation types in the Loess Plateau, China. CATENA, 158: 55-62.

Zhao C L, Shao M A, Jia X X, et al. 2017b. Estimation of spatial variability of soil water storage along the south-north transect on China's Loess Plateau using the state-space approach. Journal of Soils and Sediments, 17(4): 1009-1020. 